DOI $10.35381 / \mathrm{cm} . v 7 i 3.568$

\title{
Retos del consentimiento informado en salud de menores de edad
}

\section{Challenges of informed consent in health in minors}

\author{
Claudio Esteban Bravo-Pesántez \\ claudio.bravo@ucacue.edu.ec \\ Universidad Católica de Cuenca, Cuenca \\ Ecuador \\ https://orcid.org/0000-0001-5196-2953 \\ Ricardo Agustín Alarcón-Vélez \\ ricardo.alarcon@ucacue.edu.ec \\ Universidad Católica de Cuenca, Cuenca \\ Ecuador \\ https://orcid.org/0000-0002-1910-8527 \\ Susana Janeth Peña-Cordero \\ spena@ucacue.edu.ec \\ Universidad Católica de Cuenca, Cuenca \\ Ecuador \\ https://orcid.org/0000-0002-6526-2437 \\ Gina Fabiola Mora-Domínguez \\ gmorad@ucacue.edu.ec \\ Universidad Católica de Cuenca, Cuenca \\ Ecuador \\ https://orcid.org/0000-0001-7617-6385
}

Recibido: 15 de agosto de 2021

Aprobado: 15 de noviembre de 2021 
Claudio Esteban Bravo-Pesántez; Ricardo Agustín Alarcón-Vélez; Susana Janeth Peña-Cordero; Gina Fabiola Mora-Domínguez

\title{
RESUMEN
}

El consentimiento informado es la autorización voluntaria de un paciente competente 0 de un representante legal en los menores de edad para recibir un tratamiento $u$ intervención médica. Por lo tanto, el objetivo es exponer los desafíos del consentimiento informado en los niños mediante una revisión de la literatura existente, además de analizar reflexivamente como afrontar los mismos y mejorar la relación médico paciente, velando siempre por el máximo beneficio del paciente y sus familias. Se fundamentó desde un tipo de investigación descriptiva documental. El rechazo a tratamientos en pediatría es complicado, es una situación que requiere de una mayor capacidad para comprender los riesgos y consecuencias de tal decisión, médicos consideran una media de edad para aceptar el rechazo a los doce años.

Descriptores: Deontología; ética médica; ética de la ciencia. (Palabras tomadas del Tesauro UNESCO).

\begin{abstract}
Informed consent is the voluntary authorization of a competent patient or a legal representative in minors to receive medical treatment or intervention. Therefore, the objective is to expose the challenges of informed consent in children through a review of the existing literature, in addition to reflectively analyzing how to deal with them and improve the doctor-patient relationship, always ensuring the maximum benefit of the patient and their families It was based on a type of descriptive documentary research. The rejection of treatments in pediatrics is complicated, it is a situation that requires a greater capacity to understand the risks and consequences of such a decision, doctors consider an average age to accept the rejection at twelve years.
\end{abstract}

Descriptors: Deontology; medical ethics; ethics of science. (Words taken from the UNESCO Thesaurus). 


\section{INTRODUCCIÓN}

En la medicina actual es necesario la autorización en base a información previa, por un paciente competente o su representante en caso de menores de edad, para recibir un tratamiento médico o para participar en una investigación biomédica. Es necesario para el consentimiento informado que previamente se discutan los beneficios, riesgos y alternativas disponibles, además debe ser documentado en un formulario escrito, esto para corroborar el proceso de comunicación tan necesario entre el médico y sus pacientes (Silva et al. 2019)

El consentimiento informado puede ser implícito o explícito, este último es el más común pues permite explicar al paciente su situación en salud y la importancia de autorizar un determinado tratamiento. Una persona pueda decidir mediante el proceso del consentimiento informado, siempre y cuando cumpla con los siguientes requisitos: comprender y recordar la información, utilizar la información para tomar una decisión razonada y capacidad para comunicar esa decisión. (Silva et al. 2019)

La literatura acerca del consentimiento informado en menores de edad es escasa, la condición de menor de edad de un paciente no niega la importancia de su decisión en el ámbito de la salud, pero es necesario evaluar su capacidad de tomar decisiones. (Feinstein et al. 2018) La vulnerabilidad y la incapacidad legal en los menores de edad exige una mayor protección de sus derechos. La autorización del menor o su representante es obligatorio tanto en investigación como en la atención en salud, esto pues está normado en códigos de ética médica y legislaciones nacionales aplicables. En los menores de edad si es posible debe obtenerse el asentimiento además del consentimiento de su representante, esto normado en la Declaración de Helsinki (Reyes-Boceta \& Martínez-Casares, 2020).

Es necesarios una mayor capacitación en los médicos en temas de consentimiento informado en menores de edad, la mayoría de profesionales de la salud desconocen el tema de derechos en menores, como rechazar los tratamientos o la posibilidad de decidir 
en materia de salud, tanto en el ámbito asistencial como en el de la investigación. (Feinstein et al, 2018)

Por lo tanto, el objetivo es exponer los desafíos del consentimiento informado en los niños mediante una revisión de la literatura existente, además de analizar reflexivamente como afrontar los mismos y mejorar la relación médico - paciente, velando siempre por el máximo beneficio del paciente y sus familias.

\section{MÉTODO}

Se fundamentó desde un tipo de investigación descriptiva documental, generándose una revisión bibliográfica, considerando trabajos sobre el tema, en buscadores científicos como Pubmed, Scielo, Scopus, Google Scholar. Se seleccionaron estudios descriptivos, analíticos y reflexivos sobre el tema, se usaron palabras como "Consent", "Informed", "Minors", "Pediatric", "Capacity", y se buscaron desde el año 2015 en adelante. Se encontraron más de 50 artículos, de los cuales por su pertinencia en relación al tema se escogieron 13, a partir de los mismos se hará un análisis crítico de los resultados encontrados, referentes a los desafíos del consentimiento informado en menores de edad.

\section{RESULTADOS}

Mediante la revisión de artículos de alta calidad e impacto, identificamos los siguientes retos a la hora de comunicar la información a los padres y sus hijos. Uno de los pilares en el consentimiento informado es la entrega de información detallada al paciente (descripción del tratamiento, beneficios, riesgos y alternativas), pero en muchas ocasiones la información entregada es incompleta, lo cual puede generar preocupación en el paciente, en este caso en los padres o cuidadores del menor de edad.

En un estudio prospectivo sobre la información entregada a los padres de 50 niños que fueron sometidos a procedimientos de neurocirugía, (Silva et al. 2019), encontraron que 
un $98 \%$ de los padres entendieron el diagnóstico, la intervención y los riesgos del procedimiento, pero apenas a un $24 \%$ de los padres se les informó de tratamientos alternativos. Por otro lado, (Alahmad, 2018), en una revisión sistemática sobre los problemas éticos del consentimiento informado en oncología pediátrica, encontró que la mayoría de padres nunca discutieron en el consentimiento la posibilidad de tratamientos alternativos a los cuales podrían optar, lo cual generó desconfianza e incertidumbre. En secuencia, (Glaser et al. 2020), realizaron una encuesta electrónica a médicos anestesiólogos sobre la advertencia dada por organismos de Estados Unidos de avisar en el consentimiento informado, que la anestesia repetida por varias ocasiones puede generar lesión cerebral permanente en niños. Se obtuvieron 548 encuestas, donde se encontró que solo un $25 \%$ advertía sobre el riesgo de lesión cerebral permanente en el consentimiento informado, esto refleja que la información se entrega de manera incompleta lo cual afecta al proceso del consentimiento informado y al derecho a la veracidad que tienen los pacientes o sus familiares.

\section{Comprensión de la información entregada}

Para cualquier tratamiento o procedimiento es necesario dar la información necesaria, pero el consentimiento además requiere que esta sea entendida por el paciente, en los menores de edad es vital que sus padres o representantes comprendan la información, pues deben tomar una decisión informada, pero en la mayoría de ocasiones no se evalúa la comprensión sino exclusivamente la cantidad de información que se da, esto da como resultado confusión y dudas a la hora de tomar una decisión.

Por otro lado, (Feinstein et al. 2018), en una revisión de 22 artículos sobre el consentimiento informado en Anestesiología en pacientes pediátricos, encontró que la mayoría de médicos entregaba información completa a los padres antes de los procedimientos, está información era recordada pero muy poco entendida, por lo tanto, se enfatiza en la necesidad de reforzar la comprensión de los padres para una mejor 
decisión informada. Para esto se deben usar estrategias para mejorar el recuerdo y la comprensión, por ejemplo, los recursos multimedia como vídeos o imágenes en el momento de realizar el proceso del consentimiento informado.

Así mismo, (Tait \& Hutchinson, 2018), expresan en su trabajo que las personas que no entienden son más propensas a malinterpretar los riesgos y beneficios y, por lo tanto, no puede tomar decisiones efectivas, además es probable que no cumplan los tratamientos de manera adecuada, entonces es importante que el médico encuentre los mecanismos que aseguren que el paciente recibe y comprende la información que se le da.

En complemento, (Alahmad, 2018), en una revisión sistemática encontró que, en 8 de 13 estudios en investigación de oncología pediátrica, los padres no comprendieron la información, esto se explicaba por la urgencia de iniciar los tratamientos y el miedo que tenían los padres a que sus hijos no reciban los mismos o que puedan desagradar al médico con sus dudas.

A pesar de que se informe sobre un procedimiento o para una investigación la compresión es pobre por parte de los padres, sobre todo había problemas en comprender los riesgos específicos y que el proceso debía ser voluntario. Ciertas situaciones en medicina generan una dificultad para la comprensión de la información y esto explica porque no se tiene un verdadero consentimiento informado. (Murtha \& Faustino, 2020).

\section{Rechazo de tratamientos}

El rechazo a tratamientos en pediatría es complicado, es una situación que requiere de una mayor capacidad para comprender los riesgos y consecuencias de tal decisión, médicos consideran una media de edad para aceptar el rechazo a los doce años y que esta situación la han experimentado alguna vez en su carrera profesional (Feinstein et al, 2018).

En otro orden, (Kabiri et al. 2019), en su estudio en un hospital pediátrico en Irán 
encontró al encuestar a 40 padres que durante el proceso del consentimiento informado nunca se explicó sobre las consecuencias de rechazar un tratamiento para sus hijos, esto generaba malestar pues consideraban que era necesaria esta información para una mejor toma de decisiones.

Asi mismo, (Khabour et al. 2017), realizaron un estudio descriptivo y cualitativo en Jordania, dirigido a comprender las percepciones de los padres sobre el consentimiento informado y el proceso de asentimiento en la investigación con niños. La mayoría de los padres enfatizaron y estuvieron de acuerdo en la importancia de buscar la aprobación de los padres y los hijos para participar en investigaciones, reconocieron que se debe respetar la decisión del niño en el caso de que rechacen los tratamientos, pero que intentaría convencerlo de participar en la investigación, algunos mencionaron que lo obligarían a participar, pues en la cultura árabe los hijos deben respetar las decisiones de los padres, reflejando persistencia de posturas paternalistas influenciadas por las creencias o tradiciones.

\section{Influencia de la angustia en las decisiones de los padres}

La enfermedad es una situación que ocasiona gran sufrimiento en el ser humano, en la actualidad los avances en medicina permiten tratar la mayoría de padecimientos, pero muchos de los tratamientos son complejos o con una gran cantidad de riesgos o efectos adversos, esto genera angustia en el paciente e influye en su decisión, esto puede ocasionar decisiones incorrectas. Ningún padre, de manera intencional causa daño, pero la angustia de la enfermedad de sus hijos puede ocasionar Maleficencia en esto últimos. El médico debe combatir esta angustia usando varias estrategias, el consentimiento informado es una de ellas, pues mediante la información buscamos el beneficio de los menores de edad. (Guadarrama-Orozco et al, 2015).

La angustia afecta la memoria, un padre puede describir la enfermedad de su hijo, pero puede olvidar que firmo un consentimiento para un ensayo clínico. Una alternativa para 
mejorar la compresión en situaciones conflictivas es el consentimiento diferido, aquí se podría empezar a iniciar el tratamiento o investigación sin necesidad de obtener una autorización de los padres y posteriormente proceder a explicar lo realizado, al principio esto puede generar molestia, pero si lo hacemos correctamente los padres entenderán porque se procedió de esa manera. (Murtha \& Faustino, 2020)

Por consiguiente, (Alahmad, 2018), en su estudio encontró que la angustia ocasionaba que los padres no escucharan la información dada y además accedían al ensayo por no tener más alternativas, la ansiedad se asocia con una mayor necesidad de información, si se da mayor información esta ansiedad puede disminuir, incluido tanto en padres como en niños. Esto demuestra que ante situaciones delicadas que generan sufrimiento y temor en los padres, se podrían tomar decisiones en un contexto inadecuado del proceso del consentimiento informado, para evitar esta situación se debe invitar a realizar preguntas para despegar dudas, acompañar a la familia y el paciente, y ante todo buscar el beneficio del paciente como fin último, o se puede optar por un consentimiento diferido para una mejor toma de decisiones (Murtha \& Faustino, 2020)

\section{Formación en ética - comunicación en los profesionales de la salud}

Aunque en pregrado los médicos reciben formación en temas de ética profesional, donde se habla constantemente de la importancia del consentimiento informado, existe desconocimiento o se le da poca importancia al mismo, esto ha ocasionado en reiteradas ocasiones malestar en los pacientes o sus familiares e incluso acciones legales que han afectado a varios médicos, por lo tanto, es necesario enfatizar en una mejor formación ética en los futuros profesionales, otro de los problemas frecuentes es la falta de habilidades de comunicación que son vitales para un buen proceso del consentimiento informado.

Concomitantemente, (Murtha \& Faustino, 2020), en su trabajo mencionan como barreras para el cumplimiento de un buen consentimiento informado por parte de los médicos el 
poco interés y la falta de capacitaciones en este acápite que pudieran brindar los hospitales, además se menciona que los médicos más jóvenes por inexperiencia no obtienen el consentimiento informado en varios procedimientos, lo cual puede tener consecuencias legales en relación a la responsabilidad profesional. Recientemente, el interés en el consentimiento informado ha aumentado entre los profesionales médicos debido al desarrollo de nuevas directrices para médicos, por lo tanto, la formación y capacitación es necesaria para un óptimo consentimiento informado (Kabiri et al, 2019) En algunos casos se ha tratado de mejorar la formación ética en los hospitales, Sherratt et al. (2020) realizaron un estudio de tipo cualitativo para obtener más pacientes que participen en el estudio CONTRACT (ensayo clínico para comparar en niños con apendicitis aguda el tratamiento quirúrgico versus el manejo expectante en hospitales pediátricos de Reino Unido), el objetivo fue mejorar las habilidades de comunicación durante el consentimiento informado.

Los médicos presentan problemas en la comunicación, usan términos complejos que no entienden los pacientes o no toman en cuenta las preferencias de los pacientes, en investigación esto ocasiona dificultad para encontrar participantes. En este estudio se implementaron estrategias educativas en los investigadores (uso de términos claros, tomar en cuenta las preferencias de los pacientes, involucrar a los menores de edad en las decisiones), se encontró que el reclutamiento paso de un $38 \%$ a un $62 \%$, esto demuestra que la comunicación es clave en el proceso del consentimiento informado.

\section{Capacidad para decidir y participación del menor de edad en la toma de decisiones} Aunque el consentimiento informado requiere para su proceso de competencia legal y capacidad para decidir, se debe enfatizar en que el menor de edad participe activamente en este proceso. El personal de salud piensa frecuentemente que los menores de edad no deben por su condición participar del proceso de comunicación en salud, pero en varios estudios se ha corroborado que tienen la capacidad para comprender y tomar 
Claudio Esteban Bravo-Pesántez; Ricardo Agustín Alarcón-Vélez; Susana Janeth Peña-Cordero; Gina Fabiola Mora-Domínguez

decisiones en conjunto con sus padres o cuidadores. La condición de menor de edad no es una excusa en salud para negar la información, por lo tanto, el médico debe reconocer este derecho y ejecutarlo (Feinstein et al, 2018).

En su estudio, (Silva et al. 2019), encontraron que solamente en 4 de 42 casos analizados el menor de 16 años participo en la toma de decisiones, pero no se documentó la capacidad para consentir del menor de edad, esto demuestra que el personal de salud no les da la importancia debida a los menores de edad en el proceso del consentimiento informado. (Boceta et al. 2020), en su estudio encontraron que mediante la aplicación de la entrevista semiestructurada McCarthur, niños mayores de 12 años tenían capacidad para decidir sobre algunas cuestiones en salud y que incluso niños más pequeños pueden entender información sanitaria, por lo tanto, el personal de salud debe valorar la capacidad del menor e incluirlo activamente en la toma de decisiones, conjuntamente con sus padres o representantes legales.

El consentimiento informado demostró ser un medio apropiado para fortalecer la moral y estructurar al niño. No se puede determinar con exactitud cuando el menor de edad ya tiene autonomía para decidir por su propia cuenta en tema de salud o enfermedad. El desarrollo moral no es una limitación en el consentimiento informado, debe ser tomada en cuenta para hacer válida su autonomía y superar el paternalismo médico. Factores externos como la familia o las costumbres debe ser tomados en cuenta para fortalecer la moral de los niños y su autonomía. La madurez de un niño está relacionada con su experiencia con la enfermedad al reconocer los riesgos y beneficios en línea con sus emociones negativas o positivas, que estructuran el carácter de un niño, que podría ser percibido por el médico como autonomía. (Díaz-Pérez et al. 2020).

Los padres tienen un rol de protector al firmar un consentimiento, pero el objetivo debe ser fortalecer el papel del niño para desarrollar su autonomía. La moral de un niño depende de características individuales, comprensión de los hechos y experiencia con la enfermedad. Esto explica porque niños que tiene enfermedades crónicas como la 
diabetes tienen un elevado nivel de comprensión, pudiendo tomar sus propias decisiones, por lo tanto, la capacidad para decidir no es una cualidad de todo o nada, sino de niveles, un mismo paciente puede ser capaz de decidir ciertas cuestiones y ser incapaz en otras (Sherratt et al, 2020).

El asentimiento, es una afirmación de participar en una investigación o en la toma decisiones en salud, por parte de alguien que es demasiado joven para dar su consentimiento legal pero lo suficientemente maduro como para comprender el estudio. (Khabour et al, 2017). Las leyes señalan que se debe respetar la autonomía de los niños y que se les debe invitar en los procesos de decisión, la paradoja es concretar a qué edad los niños pueden participar. Sin embargo, la capacidad neurológica madura no significa automáticamente que un niño es competente para cualquier decisión médica, el médico debe valorar exhaustivamente la capacidad del menor de edad, permitiendo según el caso su participación. Entre 12 a 18 años lo mejor es un consentimiento doble, que da u $\mathrm{n}$ beneficio judicial y ético. Según la capacidad que tenga el menor de edad para decidir, este debe estar cada vez más informado e involucrado en las decisiones médicas, recordando que la autonomía tiene valor de derecho, reconocido hacia los pacientes (Grootens-Wiegers et al, 2017).

\section{CONCLUSIONES}

El consentimiento informado es un proceso conocido y realizado por la gran mayoría del personal de salud, pero presenta algunos retos en los menores de edad, encontramos que los padres en la mayor parte de casos entendían la información dada por el médico $\mathrm{u}$ investigador, pero sentían que algunos datos como los riesgos o tratamientos alternativos eran explicados brevemente o incluso no se les informaba de los mismos, en el tema de que los menores de edad puedan participar en la toma decisiones se encontró que la mayoría de padres estaban de acuerdo pero en situaciones conflictivas como en investigaciones biomédicas, preferían ellos tomar las decisiones por considerar 
que los menores no entenderían la información. En el tema de rechazo de tratamientos se encontró que la mayoría de padres respetarían esa decisión, pero que intentarían convencerle de que acepte el tratamiento, esto basándose en que los padres siempre buscan los mejor para sus hijos.

En la actualidad los pacientes o sujetos que participan en investigaciones necesitan ser informados para autorizar un tratamiento u intervención, el consentimiento informado es reconocido como un derecho y un imperativo en el ejercicio profesional, presenta varios retos para su uso adecuado, más aún en menores de edad que carecen de competencia legal para tomar sus propias decisiones.

Uno de los principales obstáculos al revisar la literatura, es el desconocimiento o poca importancia que le da el médico a las opiniones o preferencias de los menores de edad y la falta de comunicación para poder informar apropiadamente, esto ocasiona malestar e incluso denuncias por considerar una falta de responsabilidad hacia el menor de edad y sus familiares. Por lo tanto, se debe enfatizar en estrategias para mejorar la entrega del consentimiento informado y la comunicación por parte de los médicos, esto ha demostrado lograr menos quejas y reproches, además de mejorar la relación médica paciente.

La medicina actual exige una doble responsabilidad ética - jurídica a los médicos, el consentimiento informado es el puntal para un ejercicio eficaz y eficiente, conocer sus retos, en este caso en menores de edad, es vital para maximizar los beneficios y minimizar los daños, es obligación de las futuras generaciones y la actuales de médicos mejorar en su formación ética, buscando ante todo lo mejor para el paciente. 


\section{REFERENCIAS CONSULTADAS}

Alahmad G. (2018). Informed Consent in Pediatric Oncology: A Systematic Review of Qualitative Literature. Cancer control: journal of the Moffitt Cancer Center, 25(1), 1073274818773720. https://doi.org/10.1177/1073274818773720

Díaz-Pérez, A., Navarro Quiroz, E., \& Aparicio Marenco, D. E. (2020). Moral structuring of children during the process of obtaining informed consent in clinical and research settings. BMC medical ethics, 21(1), 122. https://doi.org/10.1186/s12910-020-00540-z

Feinstein, M. M., Pannunzio, A. E., Lobell, S., \& Kodish, E. (2018). Informed Consent in Pediatric Anesthesia: A Narrative Review. Anesthesia and analgesia, 127(6), 1398-1405. https://doi.org/10.1213/ANE.0000000000003705 Fernandez, Allison M., Scott C. Watkins, David J. Clendenin, Erik B. Smith, Jenny E. Dolan, Ernest Amankwah, Ali Jalali, et al. 2020. "A Survey of Current Practices of Informed Consent by Pediatric Anesthesiologists". Paediatric Anaesthesia 30 (7): 835-37.

Glaser, J., Nouri, S., Fernandez, A., Sudore, R. L., Schillinger, D., Klein-Fedyshin, M., \& Schenker, Y. (2020). Interventions to Improve Patient Comprehension in Informed Consent for Medical and Surgical Procedures: An Updated Systematic Review. Medical decision making : an international journal of the Society for Medical Decision Making, 40(2), 119-143. https://doi.org/10.1177/0272989X19896348

Grootens-Wiegers, P., Hein, I. M., van den Broek, J. M., \& de Vries, M. C. (2017). Medical decision-making in children and adolescents: developmental and $\begin{array}{lll}\text { neuroscientific aspects. BMC } & \text { pediatrics, 17(1), }\end{array}$ https://doi.org/10.1186/s12887-017-0869

Guadarrama-Orozco, J. H., Garduño Espinosa, J., Vargas López, G., \& Viesca Treviño, C. (2015). Consentimiento informado y rechazo de los padres al tratamiento médico en edad pediátrica. El umbral de la tolerancia médica y social. Parte II [Informed consent and parental refusal for medical treatment in childhood. The threshold of medical and social tolerance. Part II]. Boletin médico del Hospital Infantil de Mexico, 72(4), 284-289. https://doi.org/10.1016/j.bmhimx.2015.07.002 
Kabiri, N., Hajebrahimi, S., Alizadeh, G., Azimzadeh, S., Farajzadeh, N., \& Talebpour, A. (2019). Promoting informed consent in a children's hospital in Tabriz, Iran: a best practice implementation project. $\mathrm{JBI}$ database of systematic reviews and implementation reports, 17(12), 2570-2577. https://doi.org/10.11124/JBISRIR-D$\underline{19-00060}$

Khabour, O. F., Alomari, M. A., \& Al-Sheyab, N. A. (2017). Parental Perceptions About Informed Consent/Assent in Pediatric Research in Jordan. Journal of empirical research on human research ethics : JERHRE, 12(4), 261-268. https://doi.org/10.1177/1556264617718937

Murtha, T. D., \& Faustino, E. (2020). Is "Informed Consent" Truly Informed?. Pediatric critical care medicine : a journal of the Society of Critical Care Medicine and the World Federation of Pediatric Intensive and Critical Care Societies, 21(6), 589590. https://doi.org/10.1097/PCC.0000000000002242

Reyes-Boceta, O., \& Martínez-Casares, M. (2020). El consentimiento informado en el menor maduro: comprensión y capacidad de decisión [Informed consent in the mature minor: understanding and decision-making capacity]. Anales de Pediatría, 95(6), 413-422. https://doi.org/10.1016/..anpedi.2020.10.011

Sherratt, F. C., Beasant, L., Crawley, E. M., Hall, N. J., \& Young, B. (2020). Enhancing communication, informed consent and recruitment in a paediatric urgent care surgical trial: a qualitative study. BMC pediatrics, 20(1), 140. https://doi.org/10.1186/s12887-020-02040-

Silva, A., Wijesinghe, H., Mundil, N., Lo, W., Walsh, A. R., Solanki, G. A., \& Rodrigues, D. (2019). Consent in paediatric neurosurgery: adequacy of documentation and parental perspectives. Child's nervous system : ChNS : official journal of the International Society for Pediatric Neurosurgery, 35(12), 2363-2369. https://doi.org/10.1007/s00381-019-04285-

Tait, A. R., \& Hutchinson, R. J. (2018). Informed Consent Training in Pediatrics-Are We Doing Enough? JAMA pediatrics, 172(3), 211-212. https://doi.org/10.1001/jamapediatrics.2017.4088 
Revista Interdisciplinaria de Humanidades, Educación, Ciencia y Tecnología

Año VII. Vol. VII. N³. Edición Especial III. 2021

Hecho el depósito de ley: pp201602FA4721

ISSN-L: 2542-3029; ISSN: 2610-802X

Universidad Nacional Experimental Francisco de Miranda (UNEFM). Santa Ana de Coro. Venezuela

Claudio Esteban Bravo-Pesántez; Ricardo Agustín Alarcón-Vélez; Susana Janeth Peña-Cordero;

Gina Fabiola Mora-Domínguez

@2021 por los autores. Este artículo es de acceso abierto y distribuido según los términos y condiciones de la licencia Creative Commons Atribución-NoComercial-Compartirlgual 4.0 Internacional (CC BY-NC-SA 4.0)

(https://creativecommons.org/licenses/by-nc-sa/4.0/). 